\title{
Stent-assisted coil embolization of MCA aneurysm via a trans-posterior communicating artery access
}

\author{
Krishna C. Joshi, MBBS, MCh, ${ }^{1}$ Daniel F. Heiferman, MD, ${ }^{2}$ André Beer-Furlan, MD, ${ }^{1}$ \\ and Demetrius K. Lopes, MD
}

'Department of Neurological Surgery, Rush University Medical Center, Chicago; ${ }^{2}$ Department of Neurological Surgery, Loyola University Stritch School of Medicine, Maywood; and ${ }^{3}$ Cerebrovascular Neurosurgery and Comprehensive Stroke Center, Chicago, Illinois

External carotid artery (ECA) to internal carotid artery (ICA) bypass is a well-established procedure for the treatment of chronic ischemic diseases of the carotid artery. Rarely de novo aneurysms can develop at the site of anastomosis. The treatment of these aneurysms can be very challenging due to various factors, including the presence of graft, previous craniotomy, atherosclerotic vessels, and lack of direct access. In this video the authors report and discuss the management of a right middle cerebral artery (MCA) wide-necked de novo aneurysm by stent-assisted coiling through a retrograde trans-posterior communicating artery access.

The video can be found here: https://youtu.be/MBKolPvOErU.

KEYWORDS endovascular; aneurysm; trans-posterior communicating artery access; anastomosis pseudoaneurysm; stent-assisted coil embolization; video 